\title{
Bernoulli Operational Matrix of Fractional Derivative for Solution of Fractional Differential Equations
}

\author{
Rachid Belgacem ${ }^{1}$, Ahmed Bokhari ${ }^{2}$ and Abdessamad Amir ${ }^{3}$ \\ ${ }_{1,2}$ Hassiba Ben Bouali University of Chlef (UHBC), Algeria \\ ${ }^{3}$ Abdelhamid Ibn Badis University of Mostaganem (UABM), Algeria \\ ${ }^{1}$ belgacemrachid02@yahoo.fr, ${ }^{2}$ bokhari.ahmed@ymail.com, ${ }^{3}$ amirabdessamad@yahoo.fr
}

\begin{abstract}
The aim of this paper is to present a numerical method based on Bernoulli polynomials for numerical solutions of fractional differential equations(FDEs). The Bernoulli operational matrix of fractional derivatives 31 is derived and used together with tau and collocation methods to reduce the FDEs to a system of algebraic equations. Hence, the solutions obtained using this method give good approximations. Illustrative examples are included to demonstrate the validity and applicability of the proposed method.
\end{abstract}

Keywords: Bernoulli polynomials, operational matrix of fractional derivatives, Caputo derivative, fractionalorder differential equations.

2010 MSC No: 65M70, 34A08, 65M70.

\section{Introduction}

The Fractional order calculus is a generalization of ordinary differentiation and integration to arbitrary order. The idea of fractional calculus started from some speculations of G.W. Leibniz (1695-1697) and L. Euler (1730) [8]. A history of the development of fractional differential operators can be found in [8, 24, 27]. Fractional derivatives offer more accurate models of real world problems than integer derivatives. Fractional differential equations provides an excellent mathematical description for modeling many complex phenomena in different fields such as mechanics 29], biology [2, 14], chemistry [6], economics [4], control theory [28] and others.

The analytic results on the existence and uniqueness of solutions to the FDEs has been further investigated and discussed by many researchers. For more details, we refer the reader to [21, 27, 30]. In general, most of the FDEs do not have exact solutions. Particularly, there is no known method for finding exact solutions of FDEs. In order to investigate the diffuclty in obtaining analytical solution to the FDEs, many authors have proposed an approximate method for the numerical solution of FDEs, some of these methods are based on the use of operational matrices, which reduce the solution of the fractional order differential equations to the solution of algebraic equations. In order to achieve this, there are two approaches.

One approach is based on converting the underlying FDE to fractional integral equation through integration, and using the operational matrix of fractional order integration, to eliminate the integral operations. for example, Bernoulli polynomials [15], Bernoulli wavelets [20], Legendre polynomial [1], Chebyshev polynomials [5], Chebyshev wavelets [23] and Bernstein polynomials [34.

Another very effective approach for solving FDEs is based on using operational matrix of fractional derivatives, to eliminate the differential operators, in order to reducing the underlying problem into solving a system of algebraic equations. In this respect, several authors employed these operational matrices for obtaining numerical solutions of FDEs, such as, Legendre polynomials [35, Legendre wavelets [16], Bernstein polynomials [33] and Genocchi 
polynomials [17.

In the present paper, we introduce a the operational matrix of derivative based on Bernoulli polynomials 31, for solving numerically linear and non-linear FDEs, using tau and collocation methods respectively.

The paper is organized as follows, in section 2, some necessary definitions of the fractional calculus are introduced. An overview on Bernoulli polynomials, including some of their important properties, is also given in the same section together with arbitrary function approximation. In section 3 , we derive the Bernoulli operational matrix of fractional order derivative in the Caputo sense. Section 4 is devoted to the numerical method for solving the FDEs with initial and boundary value. In Section 5 the proposed methods are applied to several examples. Finally, Section 6 concludes the paper.

\section{Preliminaries and notations}

\subsection{The fractional derivative in the Caputo sense}

There are several definitions of the fractional derivative of order $\mu$. The most commonly used definitions are RiemannLiouville and Caputo. The Caputo fractional derivative uses initial and boundary conditions of integer order derivatives having some physical interpretations. Because of this specific reason, in this work we shall use the Caputo fractional derivative (see [25] and [30]):

Definition 2.1. The Caputo definition of the fractional-order derivative is

$$
D^{\mu} f(x)=\frac{1}{\Gamma(n-\mu)} \int_{0}^{x} \frac{f^{(n)}(t)}{(x-t)^{\mu+1-n}} d t, \quad n-1<\mu \leqslant n, n \in \mathbb{N},
$$

where $\mu>0$ is the order of the derivative and $n$ is the smallest integer greater than $\mu$. For the Caputo derivative we have

$$
\begin{aligned}
D^{\mu} C & =0, \quad C \text { is constant, } \\
D^{\mu} x^{q} & = \begin{cases}0, & \text { for } q \in \mathbb{N}_{0} \text { and } q<\lceil\mu\rceil, \\
\frac{\Gamma(q+1)}{\Gamma(q+1-\mu)} x^{q-\mu}, & \text { for } q \in \mathbb{N}_{0} \text { and } q \geq\lceil\mu\rceil \text { or } q \notin \mathbb{N} \text { and } q>\lfloor\mu\rfloor .\end{cases}
\end{aligned}
$$

We use the ceiling function $\lceil\mu\rceil$ to denote the smallest integer greater than or equal to $\mu$, and the floor function $\lfloor\mu\rfloor$ to denote the largest integer less than or equal to $\mu$. Moreover $\mathbb{N}=\{1,2, \ldots\}$ and $\mathbb{N}_{0}=\{0,1,2, \ldots\}$. Recall that for $\mu \in \mathbb{N}$, the Caputo differential operator coincides with the usual differential operator of an integer order. Similar to the integer-order differentiation, the Caputo fractional differentiation is a linear operation

$$
D^{\mu}(\gamma f(x)+\delta g(x))=\gamma D^{\mu} f(x)+\delta D^{\mu} g(x),
$$

where $\gamma$ and $\delta$ are constants.

\subsection{Properties of the Bernoulli polynomials}

In this section, we recall some properties of the Bernoulli polynomials which will be of fundamental importance in the sequel. The classical Bernoulli polynomials $B_{n}(t)$ is usually defined by means of the exponential generating functions [3]

$$
\frac{x e^{x t}}{e^{x}-1}=\sum_{n=0}^{\infty} \frac{B_{n}(t) x^{n}}{n !}
$$

where $B_{n}(t)$ is the Bernoulli polynomials of degree $n$ and is given on the interval $[0,1]$ by $[9$

$$
B_{n}(t)=\sum_{i=0}^{n}\left(\begin{array}{c}
i \\
n
\end{array}\right) \alpha_{n-i} t^{i}
$$

where $\alpha_{i}=B_{i}(0), i=0,1, \ldots, n$ are Bernoulli numbers. These numbers produce the following exponential generating function 3 ]

$$
\frac{t}{e^{t}-1}=\sum_{i=0}^{\infty} B_{i}(0) \frac{t^{i}}{i !}
$$


The first Bernoulli numbers are

$$
\alpha_{0}=1, \alpha_{1}=-\frac{1}{2}, \alpha_{2}=-\frac{1}{6}, \cdots,
$$

with $\alpha_{2 i+1}=0, i=1,2,3, \cdots$, and the first Bernoulli polynomials are

$$
B_{0}(t)=1, B_{1}(t)=t-\frac{1}{2}, B_{2}(t)=t^{2}-t-\frac{1}{6}, B_{3}(t)=t^{3}-\frac{3}{2} t^{2}+\frac{1}{2} t, \cdots
$$

The Bernoulli polynomials satisfy the following relations [12]:

$$
\begin{gathered}
B_{n}(0)=B_{n}(1)=\alpha_{n}, n \neq 1, \\
B_{n}^{\prime}(t)=n B_{n-1}(t), n \geq 1, \\
\int_{0}^{1} B_{n}(t) \mathrm{d} t=0, n \geq 1,
\end{gathered}
$$

Another interesting property that they satisfy is given by [3]:

$$
\int_{0}^{1} B_{n}(t) B_{m}(t) \mathrm{d} t=\frac{(-1)^{n+m}}{\left(\begin{array}{c}
n+m \\
n
\end{array}\right)} \alpha_{n+m}, \quad n, m \geq 1 .
$$

According to 22, Bernoulli polynomials form a complete basis over the interval [0,1]. If we introduce the Bernoulli vector $B(t)$ in the form

$$
B(t)^{T}=\left[B_{0}(t), B_{1}(t), \ldots, B_{N}(t)\right],
$$

then the derivative of $B(t)$, with the aid of (7), can be expressed in the matrix form by

$$
\underbrace{\left[\begin{array}{c}
B_{0}^{\prime}(t) \\
B_{1}^{\prime}(t) \\
B_{2}^{\prime}(t) \\
\vdots \\
B_{N-1}^{\prime}(t) \\
B_{N}^{\prime}(t)
\end{array}\right]}_{B^{\prime}(t)}=\underbrace{\left[\begin{array}{ccccccc}
0 & 0 & 0 & \cdots & 0 & 0 & 0 \\
1 & 0 & 0 & \cdots & 0 & 0 & 0 \\
0 & 2 & 0 & \cdots & 0 & 0 & 0 \\
\vdots & \vdots & \vdots & \ddots & \vdots & \vdots & \vdots \\
0 & 0 & 0 & \cdots & N-1 & 0 & 0 \\
0 & 0 & 0 & 0 & 0 & N & 0
\end{array}\right]}_{\mathbf{D}^{(1)}} \underbrace{\left[\begin{array}{c}
B_{0}(t) \\
B_{1}(t) \\
B_{2}(t) \\
\vdots \\
B_{N-1}(t) \\
B_{N}(t)
\end{array}\right]}_{B(t)}
$$

where $\mathbf{D}^{(1)}$ is the $(N+1) \times(N+1)$ Bernoulli polynomials operational matrix of derivative.

\subsection{Function approximation in terms of Bernoulli Polynomials}

Suppose that $H=L^{2}([0,1])$ and $\left\{B_{0}(t), B_{1}(t), \ldots, B_{N}(t)\right\} \subset H$ be the set of Bernoulli polynomials and $Y=$ $\operatorname{Span}\left\{B_{0}(t), B_{1}(t), \ldots, B_{N}(t)\right\}$. Since $Y$ is a finite dimensional subspace, $Y$ is a complete subspace of $H$ [22. Thus for an arbitrary element $g(t)$ in $H$, there exists a unique best approximation $(t) \in Y$ such that

$$
\forall y(t) \in Y,\|g(t)-\widehat{g}(t)\| \leq\|g(t)-y(t)\|
$$

This implies that

$$
\forall y(t) \in Y, \quad\langle g(t)-\widehat{g}(t), y(t)\rangle=0,
$$

where $\langle$,$\rangle denotes the inner product defined by$

$$
\langle f(t), g(t)\rangle=\int_{0}^{1} f(t) g(t) \mathrm{d} t .
$$

Since $\widehat{g} \in Y$, there exist unique vector $G^{T}=\left[g_{0}, g_{1}, \ldots, g_{N}\right]$ such that

$$
g \simeq \widehat{g}=\sum_{n=0}^{N} g_{n} B_{n}(t)=G^{T} B(t) .
$$


Using Equation (11), we get

$$
\left\langle g(t)-G^{T} B(t), B_{i}(t)\right\rangle=0, \forall B_{i}(t) \in Y,
$$

or equivalently

$$
G^{T}\langle B(t), B(t)\rangle=\langle g(t), B(t)\rangle,
$$

where $\langle B(t), B(t)\rangle=\int_{0}^{1} B(t) B^{T}(t) \mathrm{d} t$ is an $(N+1) \times(N+1)$ matrix.

Let $M=\langle B(t), B(t)\rangle$, this matrix can be calculated using Eq [8]. Therefore, any function $g(t) \in L^{2}[0,1]$ can be expanded by Bernoulli polynomials as $g(t)=G^{T} B(t)$, where

$$
G=M^{-1}\langle g(t), B(t)\rangle .
$$

\subsubsection{Error bound}

The error bounds of the truncated Bernoulli series expansion is discussed in [19]

Lemma 2.2. Suppose $f(t) \in C^{m+1}[0,1]$ and $S_{m}=\operatorname{Span}\left\{B_{0}(t), B_{1}(t), \ldots, B_{N}(t)\right\}$. If $C^{T} B(t)$ is the best approximation $f(t)$ out of $S_{m}$, then

$$
\left\|f(t)-C^{T} B(t)\right\|_{L^{2}[0,1]} \leq \frac{K}{(m+1) ! \sqrt{2 m+3}},
$$

where $K=\max _{t \in[0,1]}\left|f^{(m+1)}(t)\right|$.

\section{Bernoulli Operational Matrix of Fractional Order Derivative}

The main objective of this section is to generalize the Bernoulli operational matrix of derivatives to the fractional calculus. From Eq. 10$]$, it is clear that

$$
\frac{d^{n} B(t)}{d t}=\left(\mathbf{D}^{(1)}\right)^{n} B(t)
$$

where $n \in \mathbb{N}$ and the superscript in $\mathbf{D}^{(1)}$, denotes the matrix powers. Thus

$$
\mathbf{D}^{(n)}=\left(\mathbf{D}^{(1)}\right)^{n}, \quad n=1,2, \cdots
$$

Lemma 3.1. Let $B_{i}(t)$ be the ith Bernoulli polynomial. Then

$$
D^{\mu} B_{i}(t)=[0,0, \ldots, 0] B_{i}(t), \quad i=0,1, \ldots,\lceil\mu\rceil-1, \mu>0 .
$$

Proof. The lemma can easily be proved using Eqs. (2)-(6).

In the following theorem we derive the operational matrix of fractional order derivative for the Bernoulli polynomials.

Theorem 3.2. Let $B(t)$ be the Bernoulli vector given in (9) and suppose $\mu>0$. Then,

$$
D^{\mu} B(t) \simeq \mathbf{D}^{(\mu)} B(t),
$$

where $\mathbf{D}^{(\mu)}$ is $(N+1) \times(N+1)$ operational matrix of fractional derivative of order $\mu$ in the Caputo sense and is defined as follows

$$
\mathbf{D}^{(\mu)}=\left[\begin{array}{cccc}
0 & 0 & \cdots & 0 \\
\vdots & \vdots & \cdots & 0 \\
0 & 0 & . \cdot & 0 \\
\theta_{\lceil\mu\rceil, 0, r}^{(\mu)} & \theta_{\lceil\mu\rceil, 1, r}^{(\mu)} & \cdots & \theta_{\lceil\mu\rceil, N, r}^{(\mu)} \\
\vdots & \vdots & \cdots & \vdots \\
\sum_{r=\lceil\mu\rceil}^{i} \theta_{i, 0, r}^{(\mu)} & \sum_{r=\lceil\mu\rceil}^{i} \theta_{i, 1, r}^{(\mu)} & \cdots & \sum_{r=\lceil\mu\rceil}^{i} \theta_{i, N, r}^{(\mu)} \\
\vdots & \vdots & \ldots & \vdots \\
\sum_{r=\lceil\mu\rceil}^{N} \theta_{N, 0, r}^{(\mu)} & \sum_{r=\lceil\mu\rceil}^{N} \theta_{N, 1, r}^{(\mu)} & \cdots & \sum_{r=\lceil\mu\rceil}^{N} \theta_{N, N, r}^{(\mu)}
\end{array}\right],
$$


where $\theta_{i, j, r}^{(\mu)}$ is given by

$$
\theta_{i, j, r}^{(\mu)}=\frac{i ! \alpha_{i-r}}{(i-r) ! \Gamma(r+1-\mu)} c_{r, j},
$$

with $\alpha_{i}$ being the Bernoulli number and $c_{r, j}$ can be obtained from $(13)$. Note that in $\mathbf{D}^{(\mu)}$, the first $\lceil\mu\rceil$ rows coefficients are all equal to zero.

Proof. Using Equations (3), (4) and (6) we have

$$
\begin{aligned}
D^{\mu} B_{i}(t) & =\sum_{r=0}^{i}\left(\begin{array}{l}
i \\
r
\end{array}\right) \alpha_{i-r} D^{\mu} t^{r} \\
& =\sum_{r=0}^{i}\left(\begin{array}{l}
i \\
r
\end{array}\right) \alpha_{i-r} \frac{\Gamma(r+1)}{\Gamma(r+1-\mu)} t^{r-\mu} \\
& =\sum_{r=0}^{i} \frac{i !}{r !(i-r) !} \alpha_{i-r} \frac{\Gamma(r+1)}{\Gamma(r+1-\mu)} t^{r-\mu} \\
& =\sum_{r=0}^{i} \frac{i !}{(i-r) !} \frac{\alpha_{i-r}}{\Gamma(r+1-\mu)} t^{r-\mu}
\end{aligned}
$$

or

$$
D^{\mu} B_{i}(t)=\sum_{r=\lceil\mu\rceil}^{i} b_{i, r}^{(\mu)} t^{r-\mu}, \quad i=\lceil\mu\rceil, \ldots, N .
$$

where

$$
b_{i, r}^{(\mu)}=\frac{i ! \alpha_{i-r}}{(i-r) ! \Gamma(r+1-\mu)} .
$$

Assuming that $t^{r-\mu}$ can be expanded in terms of Bernoulli polynomials as

$$
t^{r-\mu} \simeq \sum_{j=0}^{N} c_{r, j} B_{j}(x)
$$

and substituting Eq21] in Eq (20) for $j=0,1, \ldots, N$, we get

$$
D^{\mu} B_{i}(x) \simeq \sum_{r=\lceil\mu\rceil}^{i} b_{i, r}^{(\mu)} \sum_{j=0}^{N} c_{r, j} \beta_{j}(x)=\sum_{j=0}^{N}\left(\sum_{r=\lceil\mu\rceil}^{i} \theta_{i, j, r}^{(\mu)}\right) \beta_{j}(x),
$$

where

Equation 222) can be rewritten as

$$
\theta_{i, j, r}^{(\mu)}=b_{i, r}^{(\mu)} c_{r, j}
$$

$$
D^{\mu} B_{i}(t) \simeq\left[\sum_{r=\lceil\mu\rceil}^{i} \theta_{i, 0, r}^{(\mu)}, \sum_{r=\lceil\mu\rceil}^{i} \theta_{i, 1, r}^{(\mu)}, \ldots, \sum_{r=\lceil\mu\rceil}^{i} \theta_{i, N, r}^{(\mu)}\right] B(t), i=\lceil\mu\rceil, \ldots, N .
$$

Finally, combining Lemma 3.1 and relations 23 , leads to the desired result.

Remark 3.3. If $\mu \in \mathbb{N}$, then Theorem 3.2 gives the same result as Eq.10.

\section{The numerical method}

In this section, we we apply the Bernoulli operational matrix of fractional derivative for solving linear and non-linear fractional differential equations. For the existence, uniqueness and continuous dependence of the solution of the problem see [10]. 


\subsection{Linear fractional differential equation}

Consider the linear Caputo fractional differential equations of the form

$$
D^{\mu} y(t)=a_{k} D^{\alpha_{k}} y(t)+a_{k-1} D^{\alpha_{k-1}} y(t)+\cdots+a_{1} D^{\alpha_{1}} y(t)+a_{0} y(t)+g(t), t \in(0, L)
$$

subject to the initial conditions

$$
y^{(i)}(0)=y_{i}, \quad i=0,1, \ldots, m-1,
$$

where $a_{0}, a_{1}, \ldots, a_{k}$ are real constant coefficients and also $\left.\mu \in\right] m-1, m\left[, 0<\alpha_{1}<\alpha_{2}<\ldots<\alpha_{k}<\mu\right.$. Here $D^{\mu}$ is the derivative of $y$ of order $\mu$ in the sense of Caputo fractional differential operator. Moreover, $y(t)$ is an unknown function of the independent variable $t$. The values of $y_{i}, i=0, \ldots, m-1$ describe the initial state of $y(t)$, and $g(t)$ is a given source function.

Now, we approximate $y(t)$ and $g(t)$ with the Bernoulli polynomial as

$$
\begin{aligned}
& y(t) \simeq \sum_{i=0}^{N} c_{i} B_{i}(t)=C^{T} B(t) . \\
& g(t) \simeq \sum_{i=0}^{N} G_{i} B_{i}(t)=G^{T} B(t) .
\end{aligned}
$$

where the vector $G$ is known (can be obtained from (13)) and is described in subsection 2.3 , but $C^{T}=\left[c_{0}, c_{1}, \ldots, c_{N}\right]$ is an unknown vector. In virtue of Theorem 3.2 and Eq. (26), the fractional state rates $D^{\mu} y(t)$ and $D^{\alpha_{j}} y(t)$ can be approximated as

$$
D^{\mu} y(t) \simeq C^{T} \mathbf{D}^{(\mu)} B(t),
$$

and

$$
D^{\alpha_{j}} y(t) \simeq C^{T} \mathbf{D}^{\left(\alpha_{j}\right)} B(t), \quad j=1, \ldots, k .
$$

From Eqs.26)-29, the residual $R(t)$ for Eq.24 can be written as

$$
R(t)=\left[C^{T}\left(\mathbf{D}^{(\mu)}-\sum_{j=1}^{k} a_{j} \mathbf{D}^{\left(\alpha_{j}\right)}-a_{0} I_{N+1}\right)-G^{T}\right] B(t)=0
$$

where $I_{N}$ denotes the identity matrix of dimension $N+1$. As in a typical tau method [7] , we can generate $N-m+1$ linear equations by applying

$$
\left\langle R(t), B_{i}(t)\right\rangle=\int_{0}^{L} R(t) B_{i}(t) \mathrm{d} t=0, \quad i=0, \ldots, N-m .
$$

Moreover, substituting Eqs. (14) and 26) into Eq. (25), we obtain

$$
y^{(i)}(0)=C^{T} \mathbf{D}^{(i)} B(0)=y_{i}, \quad i=0,1, \ldots, m-1 .
$$

Eqs. (31) and (32) generate $(N-m+1)$ and $m$ linear equations, respectively. These linear equations can be solved for unknown coefficients of the vector $C$. Consequently, $y(t)$ given in Eq. 26) can be calculated and gives a solution of Eq. 24] with the initial conditions 225].

\subsection{Non-linear fractional differential equation}

Consider the following non-linear fractional-order differential equation (NFDE):

$$
D^{\mu} y(t)=F\left(t, y(t), D^{q_{1}} y(t), \ldots, D^{q_{k}} y(t)\right)
$$

subject to the initial conditions 32 . Where $m-1<\mu \leq m, 0<q_{1}<q_{2}<\ldots<q_{k}<\mu$. It is to be noted here that $\mathrm{F}$ can be non-linear in general.

We approximate $y(t), D^{\mu} y(t)$ and $D^{q_{j}} y(t)$ for $j=1, \ldots, k$ as in the previous section, hence the residual $\bar{R}_{N}(t)$ of Eq. (33) is given by:

$$
\bar{R}(t)=C^{T} \mathbf{D}^{(\mu)} B(t)-F\left(t, C^{T} B(t), C^{T} \mathbf{D}^{\left(q_{1}\right)} B(t), \ldots, C^{T} \mathbf{D}^{\left(q_{k}\right)} B(t)\right),
$$


where $C^{T}=\left[c_{0}, c_{1}, \ldots, c_{N}\right]$ is an unknown vector. To find the solution of (33), we first calculate Eq. (34) at $N-m+1$ points. In terms of order the optimal choice is to let the collocation points be the $N-m+1$ roots of shifted Legendre polynomial $P_{N-m+1}(t)[13$. We obtain

$$
\bar{R}\left(t_{i}\right)=0, \quad i=1, \ldots, N-m+1 .
$$

Eqs. (35) with (32) generate $N-1$ non-linear system of equations in the unknown expansion coefficients $C$ of dimension $(N+1)$. Any standard iteration technique, like Newton's iterative technique can be employed for solving this system and hence the approximate solution $y(t)$ can be obtained.

Remark 4.1. It is worthy to mention here that the method described above in section 4.2, can be also applied to linear fractional differential equations.

\section{$5 \quad$ Illustrative examples}

Example 5.1. We consider the following non-linear initial value problem (see [18])

$$
\begin{gathered}
D^{3} y(t)+D^{\frac{5}{2}} y(t)+y^{2}(t)=t^{4}, t \in[0,1] \\
y(0)=y^{\prime}(0)=0, y^{\prime \prime}(0)=2 .
\end{gathered}
$$

The exact solution of this problem is $y(x)=t^{2}$.

We solve the above Eq. (36) with initial condition (37), by applying the technique described in Section 4.2 with $N=3$. We approximate the solution as

$$
y(t)=\sum_{i=0}^{3} c_{i} B_{i}(t)=C^{T} B(t)
$$

thus,

$$
D^{3} y(t)=C^{T} \mathbf{D}^{(3)} B(t), D^{\frac{5}{2}} y(t)=C^{T} \mathbf{D}^{\left(\frac{5}{2}\right)} B(t) .
$$

where

$$
\begin{aligned}
\mathbf{D}^{(1)} & =\left[\begin{array}{llll}
0 & 0 & 0 & 0 \\
1 & 0 & 0 & 0 \\
0 & 2 & 0 & 0 \\
0 & 0 & 3 & 0
\end{array}\right], \mathbf{D}^{(2)}=\left[\begin{array}{llll}
0 & 0 & 0 & 0 \\
0 & 0 & 0 & 0 \\
2 & 0 & 0 & 0 \\
0 & 6 & 0 & 0
\end{array}\right], \mathbf{D}^{(3)}=\left[\begin{array}{llll}
0 & 0 & 0 & 0 \\
0 & 0 & 0 & 0 \\
0 & 0 & 0 & 0 \\
6 & 0 & 0 & 0
\end{array}\right], \\
\mathbf{D}^{\left(\frac{5}{2}\right)} & =\left[\begin{array}{rrrr}
0 & 0 & 0 & 0 \\
0 & 0 & 0 & 0 \\
0 & 0 & 0 & 0 \\
\frac{8}{\sqrt{\pi}} & \frac{32}{3 \sqrt{\pi}} & -\frac{48}{7 \sqrt{\pi}} & \frac{32}{3 \sqrt{\pi}}
\end{array}\right] \text { and } C=\left[\begin{array}{l}
c_{0} \\
c_{1} \\
c_{2} \\
c_{3}
\end{array}\right] .
\end{aligned}
$$

Therefore Eqs. (36) and (37) become

$$
\begin{gathered}
C^{T} \mathbf{D}^{(3)} B(t)+C^{T} \mathbf{D}^{\left(\frac{5}{2}\right)} B(t)+\left[C^{T} B(t)\right]^{2}-t^{4}=0 \\
C^{T} B(0)=C^{T} \mathbf{D}^{(1)} B(0)=0, C^{T} \mathbf{D}^{(2)} B(0)=2 .
\end{gathered}
$$

Using Eq. (39), we get

$$
\left\{\begin{array}{c}
c_{0}-\frac{1}{2} c_{1}+\frac{1}{6} c_{2}=0 \\
c_{1}-c_{2}+\frac{1}{2} c_{3}=0 \\
2 c_{2}-3 c_{3}=2
\end{array}\right.
$$

Now collocate Eq. $(38)$ at the the first root of shifted Legendre polynomials $t_{0}=\frac{1}{2}$, to obtain

$$
C^{T} D^{(3)} B\left(t_{0}\right)+C^{T} D^{\left(\frac{5}{2}\right)} B\left(t_{0}\right)+\left[C^{T} B\left(t_{0}\right)\right]^{2}-t_{0}^{4}=0
$$


Solving Eqs. 41 and 40 using Newton's iterative method we obtain

$$
c_{0}=\frac{1}{3}, c_{1}=1, c_{2}=1, c_{3}=0
$$

Therefore

$$
y(t)=\left[\frac{1}{3}, 1,1,0\right]\left[\begin{array}{c}
1 \\
t-\frac{1}{2} \\
t^{2}-t+\frac{1}{6} \\
t^{3}-\frac{3}{2} t^{2}+\frac{1}{2} t
\end{array}\right]=t^{2}
$$

which is the exact solution of this problem.

Example 5.2. Consider the following linear fractional initial value problem (see [11])

$$
\begin{gathered}
D^{2} y(t)+D^{\frac{1}{2}} y(t)+y(t)=g(t), \quad g(t)=t^{2}+2+\frac{8}{3 \sqrt{\pi}} t^{1.5}, \quad t \in[0,1] \\
y(0)=y^{\prime}(0)=0 .
\end{gathered}
$$

By applying the technique described in Section 4.1 for the case $N=2$, the residual of Eq.42 can be calculated by the formula:

$$
R(t)=C^{T} \mathbf{D}^{(2)} B(t)+C^{T} \mathbf{D}^{\left(\frac{1}{2}\right)} B(t)+C^{T} B(t)-G^{T} B(t)
$$

where

$$
\begin{aligned}
\mathbf{D}^{(1)} & =\left[\begin{array}{lll}
0 & 0 & 0 \\
1 & 0 & 0 \\
0 & 2 & 0
\end{array}\right], \mathbf{D}^{(2)}=\left[\begin{array}{lll}
0 & 0 & 0 \\
0 & 0 & 0 \\
2 & 0 & 0
\end{array}\right], \mathbf{D}^{\left(\frac{1}{2}\right)}=\left[\begin{array}{rrr}
0 & 0 & 0 \\
\frac{4}{3 \sqrt{\pi}} & \frac{8}{5 \sqrt{\pi}} & \frac{-8}{7 \sqrt{\pi}} \\
-\frac{4}{15 \sqrt{\pi}} & \frac{8}{7 \sqrt{\pi}} & \frac{8}{3 \sqrt{\pi}}
\end{array}\right], \\
G^{T} & =\left[\frac{7}{3}+\frac{16}{15 \sqrt{\pi}}, 1+\frac{96}{35 \sqrt{\pi}}, 1+\frac{32}{21 \sqrt{\pi}}\right] \text { and } C^{T}=\left[c_{0}, c_{1}, c_{2}\right] .
\end{aligned}
$$

If we apply the method described in Section 4.1 to Eq. (43), we find

$$
c_{0}+\frac{4}{3 \sqrt{\pi}} c_{1}+\left(2-\frac{4}{15 \sqrt{\pi}}\right) c_{2}-\left(\frac{7}{3}+\frac{16}{15 \sqrt{\pi}}\right)=0
$$

The initial conditions in Eq. 43 give

$$
c_{0}-\frac{1}{2} c_{1}+\frac{1}{6} c_{2}=0 \quad \text { and } \quad c_{1}-c_{2}=0
$$

Solving Eqs. 45 and 46 yield $c_{0}=\frac{1}{3}, c_{1}=c_{2}=1$, and consequently $y(t)=t^{2}$ which is the exact solution of this problem.

Example 5.3. Consider the following linear fractional equation

$$
D^{\alpha} y(t)+y(t)-t^{2}-\frac{2}{\Gamma(3-\alpha)} t^{2-\alpha}=0, \quad 0<\alpha<1
$$

with the initial condition

$$
y(0)=0 .
$$

Using the technique described in Section 4.1 we solve Eq. 47] with initial condition (48) for $\alpha=0.5$ and $N=2$,

$$
\begin{gathered}
c_{0}+\frac{4}{3 \sqrt{\pi}} c_{1}-\frac{4}{15 \sqrt{\pi}} c_{2}-\left(\frac{1}{3}+\frac{16}{15 \sqrt{\pi}}\right)=0 \\
\left(\frac{2}{15 \sqrt{\pi}}+\frac{1}{12}\right) c_{1}+\frac{2}{21 \sqrt{\pi}} c_{2}-\left(\frac{1}{12}+\frac{8}{35 \sqrt{\pi}}\right)=0
\end{gathered}
$$




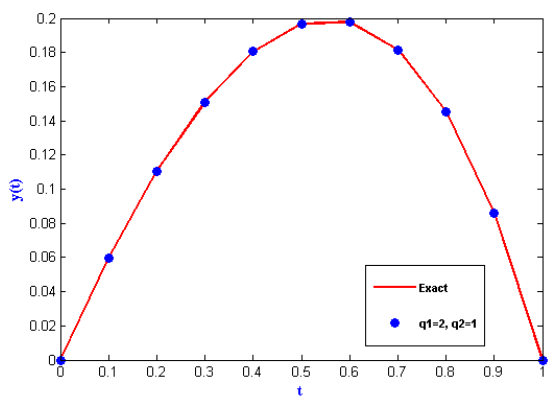

Fig. 1: Comparison of our method with $N=5$ and the exactsolution for $q_{1}=2$ and $q_{2}=1$.

\begin{tabular}{|l|l|l|l|l|l|}
\hline$N$ & 2 & 3 & 5 & 9 \\
\hline$l^{2}$ Error & $4.22 \times 10^{-02}$ & $1.47 \times 10^{-02}$ & $2.17 \times 10^{-05}$ & $2.66 \times 10^{-08}$ & $4.42 \times 10^{-11}$ \\
\hline$l^{\infty}$ Error $2.02 \times 10^{-02}$ & $7.28 \times 10^{-03}$ & $1.21 \times 10^{-05}$ & $1.52 \times 10^{-08}$ & $1.09 \times 10^{-11}$ \\
\hline
\end{tabular}

Table 1: The $l^{2}$ and $l^{\infty}$ errors for Example 4.

$$
c_{0}-\frac{1}{2} c_{1}+\frac{1}{6} c_{2}=0
$$

Solving the system of Eqs. (49), (50) and(51) we obtain

$$
y(t)=\left[\frac{1}{3}, 1,1\right]\left[\begin{array}{c}
1 \\
t-\frac{1}{2} \\
t^{2}-t+\frac{1}{6}
\end{array}\right]=t^{2},
$$

which is the exact solution.

Example 5.4. Consider the following linear boundary value problem [32]

$$
\begin{gathered}
D^{q_{1}} y(t)-D^{q_{2}} y(t)=-1-e^{t-1}, 0<t<1,1<q_{2} \leq 2,0<q_{1} \leq 1 . \\
u(0)=u(1)=0 .
\end{gathered}
$$

The exact solution of Eq. 52 with boundary condition (54) corresponding to $q_{1}=2$ and $q_{2}=1$ is $y(t)=t\left(1-e^{t-1}\right)$. In Table 1, we compare numerical results of $y(t)$ using our method with $N=6$ and $N=7$ with the exact solution and those obtained in 20, 36. Table 1 shows the $l^{2}$ and $l^{\infty}$ errors between the exact and approximate solutions for $q_{1}=2$ and $q_{2}=1$ with different values of $N$. In Figure 1 , the exact solution $y(t)$ and the approximate solution for $q_{1}=2$ and $q_{2}=1$ are plotted. Figure 2 illustrates the approximate solutions corresponding to $q_{1}=2$ and different values of $q_{2}$ near the value $1\left(q_{2}=0.7,0.8,0.9,1\right)$. Furthermore, figure 3 displays the numerical results obtained for $q_{2}=1$ and $q_{1}=1.7,1.8,1.9,2$ together with the exact solution. These Figures illustrate that series expansion of $y(t)$ has a good convergence rate.

Example 5.5. Consider the following non-linear fractional Riccati differential equation [23, [26]

$$
D^{q} y(t)=2 y(t)-y(t)^{2}+1, \quad 0<q \leq 1,
$$

with initial condition

$$
y(0)=0 .
$$

The exact solution of this problem for the case $q=1$ is

$$
y(t)=1+\sqrt{2} \tanh \left[\sqrt{2} t+\frac{1}{2} \log \left(\frac{\sqrt{2}-1}{\sqrt{2}+1}\right)\right] .
$$

This example is solved in [20] using Bernoulli wavelets method, with $k=2$ and $M=10$. It was also studied in [23] and [26] using the Chebyshev wavelet method and the modified homotopy perturbation method, respectively. Here we 


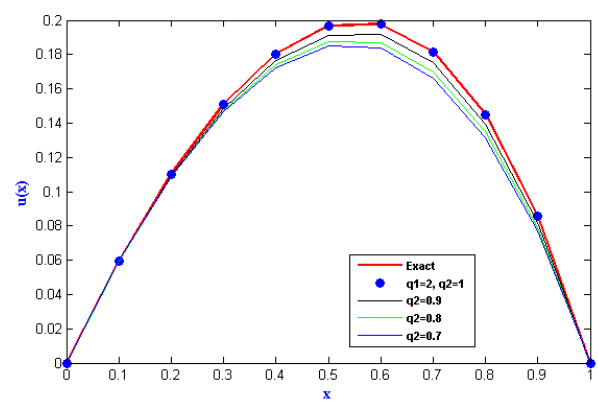

Fig. 2: Comparison of $y(t)$ for $N=6$ in the case corresponding to $q_{1}=2$ and $q_{2}=0.7,0.8,0.9,1$ with exact solution for Example 4

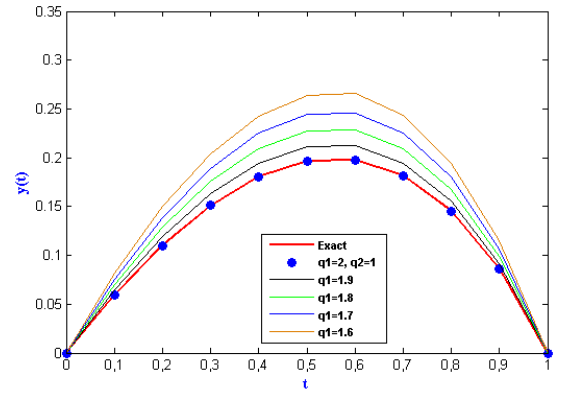

Fig. 3: Comparison of $y(t)$ for $N=6$ in the case corresponding to $q_{2}=1$ and $q_{1}=1.7,1.8,1.9,2$ with exact solution for Example 4

\begin{tabular}{|c|c|c|c|c|c|}
\hline$t$ & {$[26]$} & {$[23]$} & [20] & Our Method & $\begin{array}{c}\text { Exact solution } \\
\text { for } q=1\end{array}$ \\
\hline 0 & 0 & - & 0.000001 & 0 & 0 \\
\hline 0.1 & 0.1102940 .110311 & 0.110295 & 0.110295 & 0.110295 \\
\hline 0.2 & 0.2419650 .241995 & 0.241977 & 0.241976 & 0.241976 \\
\hline 0.3 & 0.3951060 .395123 & 0.395105 & 0.395104 & 0.395104 \\
\hline 0.4 & 0.5681150 .567829 & 0.567812 & 0.567812 & 0.567812 \\
\hline 0.5 & 0.7575640 .756029 & 0.756015 & 0.756014 & 0.756014 \\
\hline 0.6 & 0.9582590 .953576 & 0.953566 & 0.953566 & 0.953566 \\
\hline 0.7 & 1.1634591 .152955 & 1.152949 & 1.152949 & 1.152948 \\
\hline 0.8 & 1.3652401 .346365 & 1.346364 & 1.346363 & 1.346363 \\
\hline 0.9 & 1.5549601 .526909 & 1.526911 & 1.526911 & 1.526911 \\
\hline 1 & 1.7238101 .689494 & 1.689497 & 1.6894983 & 1.6894983 \\
\hline
\end{tabular}

Table 2: Numerical results for Example 5.

solve this example using the method described in section 4.2. Our results of the approximate solution with $N=5$, when $q=1$ are compared with the exact solution in Figure 4, In Table 2, we compare numerical results of $y(t)$ using our method for $N=10$ with the exact solution and those obtained in [26], 23] and [20. The absolute error for the solution $y(t)$ obtained with different values of $N=3,5,7,9,13$ of the case $q=1$ are shown in Table 2. In Figure 5 we compare the results obtained by the present approach with $N=10$, for $q=0.9,0.75,0.5$ with the exact solution, in which the figures affirm that when $q$ approaches 1 , our results approach the exact solution.

Example 5.6. Consider another fractional order Riccati differential equation [18, [20]:

$$
D^{q} y(t)=1-y(t)^{2}, \quad 0<q \leq 1,
$$

subject to the initial condition

$$
y(0)=0 .
$$

\begin{tabular}{|l|l|l|l|l|l|l|}
\hline$N$ & 2 & 4 & 6 & 10 & 12 \\
\hline$l^{2}$ Error & $9.60 \times 10^{-02}$ & $3.62 \times 10^{-03}$ & $1.32 \times 10^{-04}$ & $5.37 \times 10^{-06}$ & $1.64 \times 10^{-07}$ & $3.69 \times 10^{-8}$ \\
\hline$l^{\infty}$ Error $4.75 \times 10^{-02}$ & $1.85 \times 10^{-03}$ & $7.47 \times 10^{-05}$ & $3.03 \times 10^{-06}$ & $1.09 \times 10^{-07}$ & $2.75 \times 10^{-8}$ \\
\hline
\end{tabular}

Table 3: The $l^{2}$ and $l^{\infty}$ errors for Example 5 . 


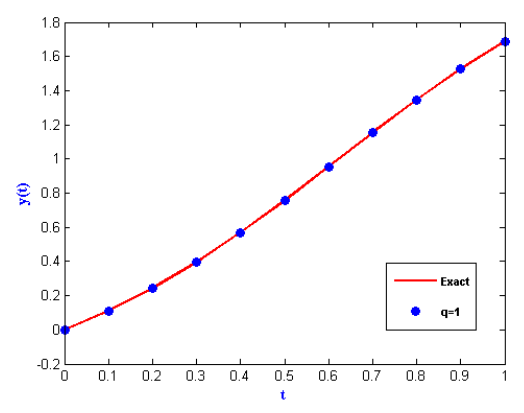

Fig. 4: Comparison of $y(t)$ for $N=5$ in the case corresponding to $q=1$ with exact solution for Example 5

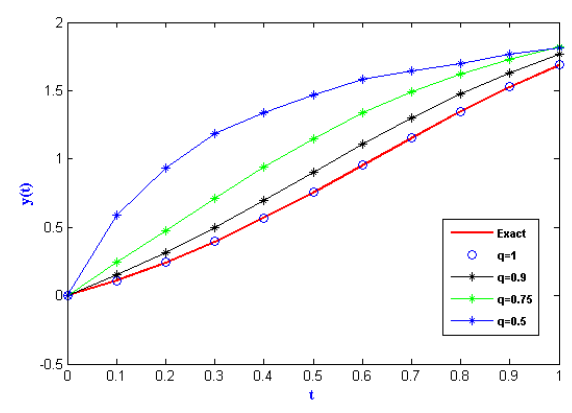

Fig. 5: Comparison of $y(t)$ for $N=10$ in the case corresponding to $q=1.7,1.8,1.9,1$ with exact solution for Example 5

\begin{tabular}{|c|c|c|c|c|}
\hline \multirow{2}{*}{$t$} & \multirow{2}{*}{ in $[20$} & \multicolumn{2}{|c|}{ Our Method } & Exaxt Solution \\
\cline { 3 - 4 } & $\mathrm{N}=5$ & $\mathrm{~N}=6$ & for $q=1$ \\
\hline 0.1 & 0.0996840 .099658 & 0.099668 & 0.099667 \\
\hline 0.2 & 0.197417 & 0.197407 & 0.197377 & 0.197375 \\
\hline 0.3 & 0.291316 & 0.291333 & 0.291310 & 0.291312 \\
\hline 0.4 & 0.3799120 .379931 & 0.379945 & 0.379948 \\
\hline 0.5 & 0.4620830 .462083 & 0.462117 & 0.462117 \\
\hline 0.6 & 0.537057 & 0.537036 & 0.537053 & 0.537049 \\
\hline 0.7 & 0.6044050 .604387 & 0.604369 & 0.604367 \\
\hline 0.8 & 0.6640520 .664061 & 0.664032 & 0.664036 \\
\hline 0.9 & 0.7162640 .716289 & 0.716296 & 0.716297 \\
\hline
\end{tabular}

Table 4: Numerical results for Example 6.

The exact solution of this system when $q=1$ is known to be 18

$$
y(t)=\frac{e^{2 t}-1}{e^{2 t}+1} .
$$

This example is solved in 18 using Legendre wavelets method, with $k=1$ and $M=25$. It was also considered in [20] by applying the Bernoulli wavelets method, with $k=2$ and $M=5$. We solve this example using the technique described in section 4.2. Our results of the approximate solution with $N=5$, when $q=1$ are compared with the exact solution in Figure 6. In Table 4, we compare numerical results of $y(t)$ using our method for $N=10$ with the exact solution and that obtained in [20]. The absolute error for the solution $y(t)$ obtained with different values of $N=2,4,6,8,12$ of the case $q=1$ are shown in Table 5 . In Figure 7 we compare the results obtained by our approach for $N=10$, when $q=0.9,0.75,0.5$ with the exact solution. From Figure 7 we see that as $q$ approaches 1 the numerical solutions converge to that of integer order differential equation.

Example 5.7. Consider the following nonlinear fractional differential equations 37

$$
\left\{\begin{array}{l}
D^{q} y_{1}(t)=-1002 y_{1}(t)+1000 y_{2}^{2}(t) \\
D^{q} y_{2}(t)=y_{1}(t)-y_{2}(t)-y_{2}^{2}(t)
\end{array}\right.
$$

\begin{tabular}{|l|l|l|l|l|l|}
\hline$N$ & 2 & 4 & 6 & 8 & 10 \\
\hline$l_{2}$ Error & $1.34 \times 10^{-02} 1.42 \times 10^{-04}$ & $7.62 \times 10^{-06}$ & $2.50 \times 10^{-07}$ & $4.26 \times 10^{-09}$ & $7.58 \times 10^{-11}$ \\
\hline$l_{\infty}$ Error $8.05 \times 10^{-02}$ & $9.10 \times 10^{-05}$ & $4.24 \times 10^{-06}$ & $1.21 \times 10^{-07}$ & $2.37 \times 10^{-09}$ & $4.11 \times 10^{-11}$ \\
\hline
\end{tabular}

Table 5: The $l^{2}$ and $l^{\infty}$ errors for Example 6 . 


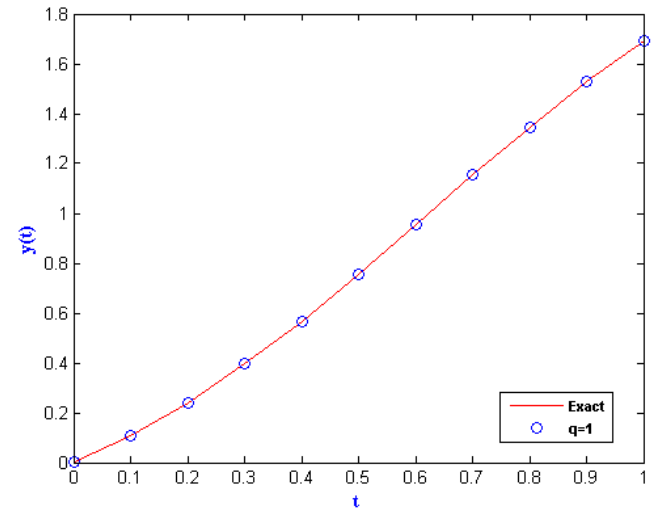

Fig. 6: Comparison of $y(t)$ for $N=5$ in the case corresponding to $q=1$ with exact solution for Example 6

Fig. 8: Comparison of $y_{1}(t)$ and $y_{2}(t)$ by our method with $N=10$ in the case corresponds to $q=0.25,0.5,0.75,1$ with exact solution for Example 7

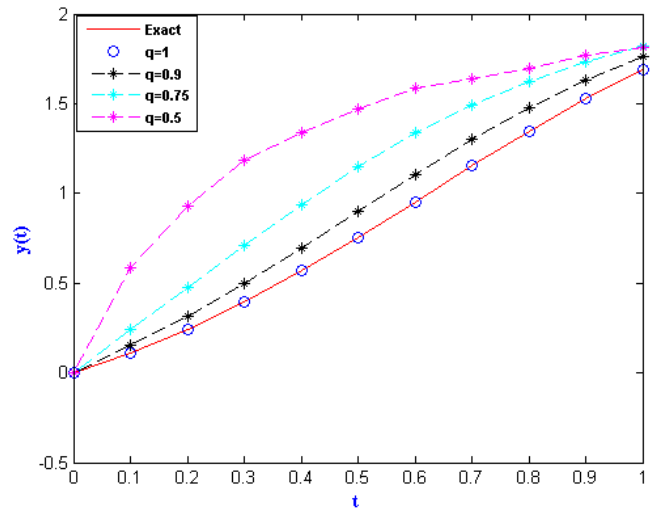

Fig. 7: Comparison of $y(t)$ for $N=10$ in the case corresponding to $q=0.5,0.75,0.9$ and 1 with exact solution for Example 6

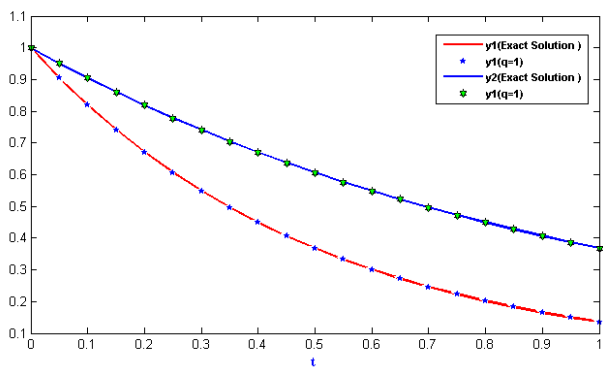

subject to the initial conditions $y_{1}(0)=1$ and $y_{2}(0)=1$. The exact solution of this system for $q=1$ is known to be $y_{1}(t)=e^{-2 t}$ and $y_{1}(t)=e^{-t}$. This example is solved by our method for $N=10$ and $q=1$. The results are compared with the exact solution $(q=1)$ in Figure 8. The results obtained when $q=0.9,0.75,0.5$ and 0.25 for $y_{1}(t)$ and $y_{1}(t)$ are plotted in Figure 9. The figures confirm that when $q$ approaches 1 our results approach the exact solution. We also compare the absolute error obtained by our method and those obtained in [37 and [17] at $t=1$ in Table 6 .

\begin{tabular}{|l|l|l|l|}
\hline$t y(t)$ & Error in [37] with $h=0.002$ & Error in [17] with $N=10$ & Our Error with $N=10$ \\
\hline $1 y_{1}(t)$ & $2.5606 \times 10^{-7}$ & $3.70730 \times 10^{-8}$ & $1.98881 \times 10^{-9}$ \\
\hline$y_{2}(t)$ & $8.0150 \times 10^{-8}$ & $2.09476 \times 10^{-11}$ & $1.99036 \times 10^{-12}$ \\
\hline
\end{tabular}

Table 6: The absolute errors obtained by the present method and that in [37] and [17] at $t=1$ for Example 7

\section{Conclusion}

In this work, a general formulation for the Bernoulli operational matrix of fractional derivative has been derived. This matrix is used to approximate numerical solution of linear and nonlinear FDEs. Our approach was based on the truncated Bernoulli polynomials expansion, Tau and collocation methods. The advantage of the present operational matrix method is that it has less computational complexity because every operational matrix of differentiation involves mostly zeros entries and thus reduces the run time and provides solutions with high accuracy. The solution obtained for these examples show that this approach can effectively solve these problems and is very simple and easy in implementation. 



Fig. 9: Comparison of $y_{1}(t)$ and $y_{2}(t)$ for $N=10$ in the case corresponds to $q=0.25,0.5,0.75,1$ with exact solution for Example 7

\section{References}

[1] M. H. Akrami, M. H. Atabakzadeh and G. H. Erjaee, The operational matrix of fractional integration for shifted Legendre polynomials, IJST (2013) 37A4: 439-444.

[2] P. Allegrini, M. Buiatti, P. Grinolini and B. J. West, Fractional Brownian motion as a nonstationary process: Analternative paradigm for DNA sequences, Phys. Rev. E, 57(4), (1998) 558-567.

https://doi.org/10.1103/physreve.57.4558

[3] G. Arfken, Mathematical Methods for Physicists, third ed., Academic press, San Diego, 1985. https://doi.org/10.1016/b978-0-12-059820-5.50004-5

[4] R. T. Baillie, M. L. King, Fractional differencing and long memory processes, J. Econometrics, 73(1), (1996) 1-3. https://doi.org/10.1016/0304-4076(95)01731-3

[5] A. H. Bhrawy, A.S. Alofi, The operational matrix of fractional integration for shifted Chebyshev polynomials, / Applied Mathematics Letters 26 (2013) 25-31. https://doi.org/10.1016/j.aml.2012.01.027

[6] J. Bisquert, Fractional diffusion in the multiple-trapping regime and revision of the equivalence with the continuous time random walk, Phys. Rev. Lett., 91(1), (2003) 1602. https://doi.org/10.1103/physrevlett.91.010602

[7] C. Canuto, M.Y. Hussaini, A. Quarteroni, T.A. Zang, Spectral Methods in Fluid Dynamics, Springer, New York, 1988. https://doi.org/10.1007/978-3-642-84108-8

[8] A. Carpinteri, F. Mainard. Fractals and Fractional Calculus in Continuum Mechanics, Springer-Verlag Wien New York, 1997. https://doi.org/10.1007/978-3-7091-2664-6

[9] F. Costabile, F. Dellaccio, M.I. Gualtieri, A new approach to Bernoulli polynomials, Rendiconti di Matematica, Serie VII 26 (2006) 1-12.

[10] K. Diethelm, N. J. Ford, Multi-order fractional differential equations and their numerical solutions, Appl. Math. Comput. 154 (2004) 621-640.

[11] E. H. Doha, A. H. Bhrawy, S. S. Ezz-Eldien, A Chebyshev spectral method based on operational matrix for initial and boundary value problems of fractional order. Comput. Math. Appl. 62 (2011), 2364 - 2373. https://doi.org/10.1016/j.camwa.2011.07.024

[12] A. Erdélyi, W. Magnus, F. Oberhettinger and F.G. Tricomi, Higher Transcendental Functions, vol. III, McGrawHill, New York, 1955.

[13] A. C. Faul, A Concise Introduction to Numerical Analysis, Boca Raton Taylor Francis 2016. https://doi.org/10.1201/b21256

[14] W. G. Glockle, T. F. Nonnenmacher, A fractional calculus approach to self-similar protein dynamics, Biophys. J., 68(1), (1995) 46-53. https://doi.org/10.1016/s0006-3495(95)80157-8 
[15] A. Golbabai, S. P. Ali Beik, An efficient method based on operational matrices of Bernoulli polynomials for solving matrix differential equations, Comp. Appl. Math, Sociedade Brasileira de Matemática Aplicada e Computacional 2014.

https://doi.org/10.1007/s40314-013-0110-y

[16] A. Isah, P. Chang, Legendre Wavelet Operational Matrix of Fractional Derivative Through Wavelet-Polynomial Transformation and its Applications in Solving Fractional Order Differential Equations, International Journal of Pure and Applied Mathematics, Volume 105 No. 1 2015, 97-114. https://doi.org/10.1063/1.4952502

[17] A. Isah, C. Phang, New operational matrix of derivative for solving non-linear fractional differential equations via Genocchi polynomials. Journal of King Saud University (2017). https://doi.org/10.1016/j.jksus.2017.02.001

[18] H. Jafari, S. Das, H. Tajadodi, Solving a multi-order fractional differential equation using homotopy analysis method, J. King Saud Univ. Sci. 23 (2011) 151-155. https://doi.org/10.1016/j.jksus.2010.06.023

[19] E. Keshavarz, Y. Ordokhani, and M. Razzaghi, A numerical solution for fractional optimal control problems via Bernoulli polynomials, Journal of Vibration and Control (2015) 1-15. https://doi.org/10.1177/1077546314567181

[20] K. Keshavarz, Y. Ordokhani, M. Razzaghi, Bernoulli wavelet operational matrix of fractional-order integration and its applications in solving the fractional-order differential equations. Appl. Math. Model. 38 (2014) 60386051. https://doi.org/10.1016/j.apm.2014.04.064

[21] A. A. Kilbas, H. M. Srivastava and J.J. Trujillo, Theory and Applications of Fractional Differential Equations. 1st Edn., Elsevier, Amsterdam, 2006. https://doi.org/10.1016/s0304-0208(06)x8001-5

[22] E. Kreyszig, Introductory Functional Analysis with Applications, John Wiley and Sons Press, New York, 1978. https://doi.org/10.2307/3616033

[23] Y. Li, Solving a nonlinear fractional differential equation using Chebyshev wavelets, Commun. Nonlinear Sci. Numer. Simul., 15 (2010) 2284 - 2292. https://doi.org/10.1016/j.cnsns.2009.09.020

[24] K. S. Miller, B. Ross: An Introduction to the Fractional Calculus and Fractional Di erential Equations, JohnWiley and Sons, Inc., New York, 1993.

[25] C. A. Monje, Y. Q. Chen, B. M. Vinagre, D. Xue, and V. Feliu, Fractional-order systems and controls, Advances in Industrial Control, Springer, 2010.

[26] Z. Odibat, S. Momani, Modified homotopy perturbation method: application to quadratic Riccati differential equation of fractional order, Chaos Solitons Fract., 36 (2008) 167-174. https://doi.org/10.1016/j.chaos.2006.06.041

[27] K.B. Oldham, J. Spanier, The Fractional Calculus: Theory and Applications of Differentiation and Integration to Arbitrary Order. Dover Publication, Mineola, 2006.

https://doi.org/10.1016/s0076-5392(09)x6012-1

[28] A. Oustaloup, La Commande CRONE. Comande Robuste D’ordre Non Entiere, Hermes Sci., Paris, 1991.

[29] A. Plonka, Recent developments in dispersive kinetics, Progr. React. Kinet. Mech., 25(2), (2000) $109-127$. https://doi.org/10.1016/s0167-7322(99)00119-1

[30] I. Podlubny, Fractional Differential Equations, An introduction to fractional derivatives, fractional differential equations, some methods of their solution and some of their applications. Academic Press, San Diego, 1999.

[31] K. Rabiei, Y. Ordokhani, E. Babolian, Numerical Solution of 1D and 2D Fractional Optimal Control of System via Bernoulli Polynomials. Int. J. Appl. Comput. Math 4: 7 (2018). https://doi.org/10.1007/s40819-017-0435-0

[32] M. Rehman, R. Ali khan, The Legendre wavelet method for solving fractional differential equations, Commun. Nonlinear Sci. Numer. Simul., 16 (2011). https://doi.org/10.1016/j.cnsns.2011.01.014

[33] D. Rostamy, M. Alipour, D. Baleanu, Solving multi-term orders fractional differential equations by operational matrices of BPs with convergence analysis. Rom. Rep. Phys. 65 (2013) 334-349. 
[34] D. Rostamy, H. Jafarib, M. Alipour, C. M. Khalique, Computational Method Based on Bernstein Operational Matrices for Multi-Order Fractional Differential Equations, Filomat 28:3 (2014), 591-601. https://doi.org/10.2298/fil1403591r

[35] A. Saadatmandi, M. Dehghan, A new operational matrix for solving fractional-order differential equations, Comput. Math. Appl. 59 (2010) 1326 - 1336. https://doi.org/10.1016/j.camwa.2009.07.006

[36] Y. G. Wang, H.F. Song, D. Li, Solving two-point boundary value problems using combined homotopy perturbation method and Green's function method. Appl. Math. Comput. 212 (2009) $366-376$. https://doi.org/10.1016/j.amc.2009.02.036

[37] X. Y. Wu, J.L. Xia, Two low accuracy methods for stiff systems. Appl. Math. Comput. 123 (2001) $141-153$. https://doi.org/10.1016/s0096-3003(00)00010-2 